\title{
Comparing diabetes mellitus risk perception among patients followed in primary and specialized healthcare: A cross-sectional study in Vaud, Switzerland
}

\author{
Francesco Gianinazzi ${ }^{*}$, Juan Ruiz², Mohamed Faouzi ${ }^{3}$, Alejandra Casillas ${ }^{4}$, Patrick Bodenmann ${ }^{5}$
}

*Correspondence: francesco.gianinazzi@chuv.ch

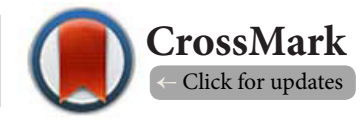

\begin{abstract}
'Service of Rheumatology, Av. Pierre-Decker 4, Hôpital Orthopédique, CHUV, 1011 Lausanne, Switzerland.
${ }^{2}$ Riviera Chablais Hospital, Site du Samaritain, Boulevard Paderewski 3, 1800 Vevey, Switzerland.

${ }^{3}$ Institut of Social and Preventive Medicine (IUMSP), Rue duBugnon 44, 1011 Lausanne, Switzerland.

4,5 Department of Ambulatory Care and Community Medicine (PMU), Rue de Bugnon 44, 1011 Lausanne, Vaud, Switzerland.
\end{abstract}

\begin{abstract}
Purpose: The purpose of this study is to 1) evaluate perceived risk for diabetes among type 2 diabetes patients with different types of diabetes follow-up: diabetologist, general practitioner, or both ("combined follow-up") in the community and university settings and 2) determine the prognostic factors for better/ higher risk perception of diabetes among these patients.

Methods: In this cross-sectional study, we quantified diabetes risk perception using Walker's RPS-DM survey. The PRIME-MD PHQ questionnaire was used to screen for mood and anxiety disorders. The global risk perception scores were coded in two categories (low/high) and compared across the various types of medical follow-up; univariate and multivariate logistic regression techniques were used to examine the association between perceived risk, patients' sociodemographic factors, diabetes characteristics and psychological profile.

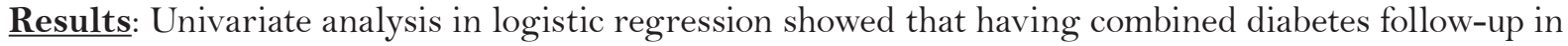
the university setting was significantly associated with higher composite risk perception $(\mathrm{OR}=14 ; 95 \% \mathrm{CI}$ 3.56-55.05) compared to patients with either single provider type follow-up. Specifically, combined followup, was linked to diabetes worry ( $\mathrm{OR}=6.45 ; 95 \% \mathrm{CI}$ 1.68-24.7), but also higher perceived risk for diabetes complications (OR=3.55; 95\% CI 1.12-11.2). These analyses also showed that perceived risk for diabetes complications was associated with longer diabetes duration (OR=1.05; 95\% CI 1.01-1.09), higher number of drug therapies $(\mathrm{OR}=1.35 ; 95 \% \mathrm{CI} 1.08-1.69)$, increased microvascular complications $(\mathrm{OR}=1.47$; 95\% CI 1.032.10) and increased vascular complications ( $\mathrm{OR}=1.31 ; 95 \% \mathrm{CI} 1.02-1.71)$.

Conclusions: Having combined follow-up in the same medical institution increases a patient's perceived risk of the complications of his/her diabetic illness. Having a more accurate self-perception of potential diabetic complications may help patients make healthier and informed lifestyle choices. Future studies should further examine the association between combined provider follow-up and risk perception, and focus on how patient-provider relationships and follow-up impact diabetic patient outcomes.
\end{abstract}

Keywords: Type 2 diabetes mellitus, behavior, risk, ambulatory care, patient care team, general practitioner

\section{Background}

Despite the increased dissemination of evidence-based guidelines for diabetes management and improvements in health systems to deliver diabetes care, there are still many diabetes patients who do not receive appropriate treatment and/or who do not seek care. Patient-centred strategies are necessary 
for outreach to these patient populations. Currently, there is considerable interest about diabetes disease risk perception among patients - a known relevant predictor of patient adherence to treatment plan [1,2]. Disease risk perception is of critical importance, as perception affects patient behaviour, which can affect diabetes outcomes and patient quality of life.

The perceived risk for type 2 diabetes is low in the general population of patients who have no known diabetes diagnosis. Relatively few patients know how to accurately bring their actual risk into line with perceived risk $[3,4]$. The difference between statistical risk and perceived risk can be accounted for by the fact that a subject's reaction to risk is most often guided by protective emotions, rather than by logical reasoning (theory of heuristics in decision-making) [5].

"Optimistic bias" is the notion of feeling protected from the risk of developing a disease compared to other individuals of the same gender and age. This has especially been observed in patients who have a high risk of developing diabetes [4]. Following this, patients who then develop diabetes often have trouble evaluating their cardiovascular risk (compared to an objective value from confirmed risk scores) [6].

There is also a low fund of knowledge of the link between cardiovascular risk factors and cardiovascular disease outcome among these patients. However, this risk is better understood among the following groups of diabetic patients- 1) women, 2) patients with a higher level of education and 3) higher income, and by 4) patients who are on cardio-protective drug treatments [7]. In addition to the misperception of cardiovascular risk, there is a tendency to underestimate the probability of a cardiovascular event when patients compare themselves to other patients in a similar clinical situation (relative risk), which can be partially explained by optimistic bias [8,9]. Other studies have also described an overestimation of absolute cardiovascular risk by diabetes patients $[6,10,11]$.

Examining perceived risk among diabetes patients may prove useful in practice; for example, a patient's fund of knowledge and predisposition for low (and inaccurate) perceived risk of their diabetes course may be linked to the way that patient healthcare is organised and delivered. The association between type of provider follow-up and patient perceived risk has not been studied thoroughly, although it has been shown that improved metabolic control is related to treatment by a multi-disciplinary point-of-care team $[12,13]$. One can speculate that perhaps patients are more educated in their disease course (and thus possess a better assessment of their risk) when cared for by a team of providers.

Our study examined the association between "type of provider" diabetes follow-up (general practitioner vs. specialist diabetologist vs. "combined"/both) and perceived composite risk of diabetes (including perceived risk for diabetic complications), as measured by validated Risk Perception Survey-Diabetes Mellitus (RPS-DM) [1]. We also examined how certain patient characteristics (well known to affect risk perception and aspects of diabetes control, like depression and diabetes duration) were related to this global measure of perceived risk $[\mathbf{1 4 , 1 5}]$. Our hypothesis was that a combined follow-up would increase the perceived risk of diabetes, and specifically a higher perceived risk of diabetic complications. We also hypothesized that worry about diabetes (a major element of perceived risk) would be associated with anxiety disorders and depression.

\section{Methods}

\section{Study design, setting and participants}

This cross-sectional study included patients who had type 2 diabetes, were capable of communicating in French (reading comprehension), and received their medical care at the Lausanne ambulatory clinic and university hospital. Between September 2010 and April 2011, we actively searched for this particular patient profile by systematically consulting all patients' appointments in all physicians' diaries of the Department of Ambulatory Care and Community Medicine (PMU) or the Endocrinology and Diabetology Service of the University Hospital of Lausanne. For all these patients we checked the medical files for a type 2 diabetes diagnosis and competence in French.Those patients whose profile corresponded to the inclusion criteria were asked by their physicians if they would agree to participate in our survey. The patients were provided with an information sheet about the study.

\section{Data collection and Primary Survey}

Each participant completed three sets of questions over the telephone (interviews were conducted by a psychologist). The primary questionnaire asked about diabetes risk perception using Walker's RPS-DM survey [1]. The original English version of the RPS-DM survey was converted to French and validated by "forward-backward translation" by our research team.

\section{Risk perception of diabetes evaluation}

The RPS-DM questionnaire [1] is unique in that it evaluates the worry related to the risk of developing diabetes-related complications, but also enables other risk perceptions to be determined and used for comparison (other diseases and environmental risk). For each item, the subjects state how strongly they agree/disagree with a statement, with four response options based on an ascending Likert scale. A cumulative score is calculated for each group of questions. Higher scores indicate increased perceived risk of diabetes (Table 1). The survey is composed of five groups of questions (which partially correspond to the dimensions of risk as defined in P. Slovic's studies $[16,17])$, for a total of 26 questions evaluating risk perception as related to diabetes and its associated complications. The questions investigate the following domains: patients' perception of control over diabetes,optimistic bias, risk perception for diseases that affect or have affected the patients (diabetes complications), worry of diabetes and environmental risk perception, and patients' knowledge regarding their diabetes. 
Table 1. Diabetes risk perception subscales and score ranges.

\begin{tabular}{lll}
\hline Diabetes risk perception subscales & $\begin{array}{l}\text { Score } \\
\text { ranges }\end{array}$ & $\begin{array}{l}\text { Low risk } \\
\text { range }\end{array}$ \\
\hline Perception of control over diabetes & $1-4$ & $1-2.99$ \\
Diabetes-related worry & $1-4$ & $1-2.99$ \\
$\begin{array}{l}\text { Optimistic bias } \\
\text { Perception of the risk related to the patient's }\end{array}$ & $1-4$ & $1-2.99$ \\
disease & $1-5$ & $1-2.99$ \\
$\begin{array}{l}\text { Diabetes risk knowledge } \\
\text { Environment risk perception }\end{array}$ & $0-5$ & $0-3$ \\
Composite risk perception & $1-4$ & $1-2.49$ \\
\hline
\end{tabular}

\section{Provider follow-up type}

Patients were recruited during visits to the Department of Ambulatory Care and Community Medicine (PMU) (17531 consultations in 2013) or to the Endocrinology and Diabetology Service of the University Hospital of Lausanne (17368 consultations in 2013). We categorized patients according to the four specific types of follow-up found in the patient's medical records:

1. (GP) General practitioner at the PMU (university primary care clinic) only

2. (DIAB) Diabetologist at the Diabetology Service of the university only

3. (C1) "Combined" follow-up by a diabetologist of the Diabetology Service of the university and by a general practitioner at the PMU

4. (C2) "Combined" follow-up by a diabetologist of the Diabetology Service of the university and by a non-PMU general practitioner (non-university primary care clinic) We also paid attention to the comparison of $\mathrm{C} 1$ follow-up to the DIAB follow-up - one interest of the study was to examine the effect of a team-based care approach within the same institution, on a patient's perceived risk of their diabetes.

\section{Patients' characteristics}

Questions about socio-demographic characteristics came from the original RPS-DM survey. As in the Walker study, these were: gender, mean age, place of birth, educational level, annual household income, and employment status [1].

Patients were also surveyed by telephone for the possible presence of any anxiety-depressive disorder using an adaptation of the English language Patient Health Questionnaire (PHQ). This diagnostic tool makes it possible to identify the primary psychiatric disorders encountered in general medicine, based on DSM-IV criteria. It has been validated in various primary care settings and populations in the United States (sensitivity 75\%, specificity 90\%) [18]. The PHQ was adapted in the French version, the PRIME-MD [19], and was used for the first time for primary care settings in France by J. Norton [20].

Lastly, clinical data about metabolic syndrome and diabetes complications were linked to each respondent and used in the analysis.

\section{Statistical analysis}

For this specific analysis, we calculated and used the composite risk perception, which incorporates all the questions, to yield a global mean score, which allows one to evaluate diabetes risk perception from a wider perspective. The risk perception scores were also coded in two categories as done in previous studies (low level=0 vs high level=1) [21]. Low level of each risk perception subscale was coded as follows: perception of control over diabetes: 1-2.99 (range 1-4), diabetes-related worry: 1-2.99, (range 1-4), optimistic bias: 1-2.99 (range 1-4), perception of the risk related to the patient's disease: 1-2.99 (range 1-5), diabetes risk knowledge: 0-3 (range 0-5), environment risk perception: 1-2.49 (range 1-4), composite risk perception: 1-2.49 (range 1-4). Univariate analysis in logistic regression was used to examine the association between perceived risk score and provider type, socio-demographic factors, diabetes characteristics, disease-related complications, and psychological profile. Provider type and associated factors to the outcomes ( $p$-value $<0.05)$ were then used in a backward procedure to fit multiple different multivariate models. The data was analysed using Stata 13 software (StataCorp. 2013. Stata Statistical Software: Release 13. College Station, TX: StataCorp LP.

\section{Results}

Patient Characteristics and Follow-up group comparisons A total of 168 patients participated in the study: 33 were followed by the ambulatory practice of the university clinic only (GP); 81 were followed by the Diabetology Service of the university clinic only (DIAB); 15 patients had a combined follow-up with the ambulatory practice and a diabetologist at the university (C1); 39 had a combined follow-up with the general practitioner from the non-university clinic and the Diabetology Service of the university clinic (C2); (Figure 1).

\section{Socio-demographic characteristics}

(Tables 2a-2c) the median (iqr) age was 62 (12.5), and participants were mostly men (73.2\%). They had a high level of education (36.3\% had been to a vocational school, and $26.8 \%$ held a tertiary qualification) and a moderately high level of income (49.4\% had an annual income $>50,000 \mathrm{CHF}$ ).

\section{Socio-demographic characteristics among different types of follow-up}

Patients of the $\mathrm{C} 1$ group, compared to the DIAB group, were younger [median (iqr) age 55 (12), versus 62 (12.5)], had a tendency to have a lower economic status (33.3\% with a 20.000-30.000 CHF annual income versus 7.4\%) and tended to be unemployed or disabled $(33.3 \%$ unemployed versus 18.5\%). Patients of the C1 group, compared to the DIAB group, had younger diabetes (7 years versus 13 years), and more depressive disorders (13.3\% with major depressive disorder versus $1.2 \%, 13.3 \%$ with minor depressive disorder versus $8.6 \%$ ). 


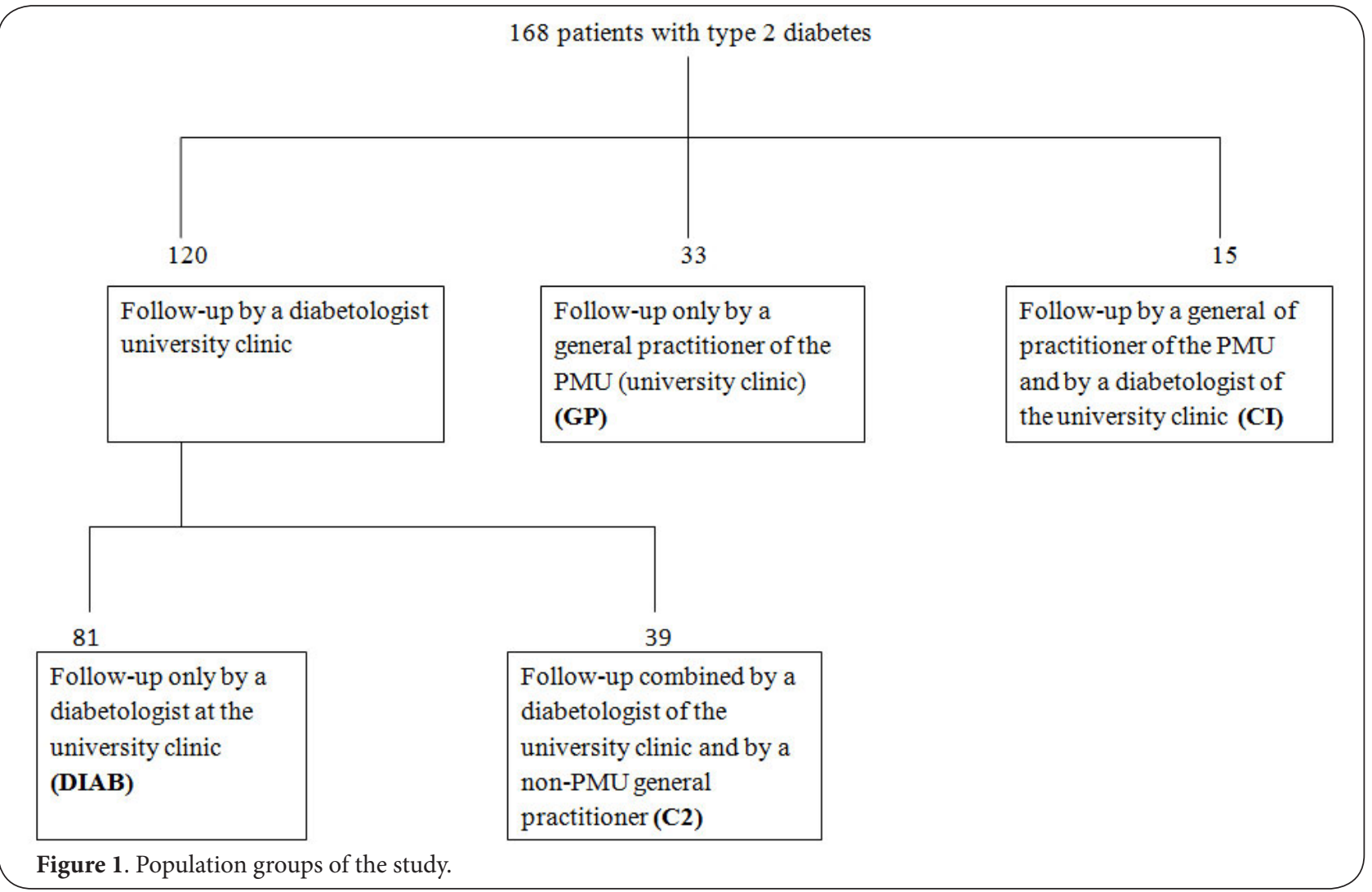

Table 2a. Patient's socio-demographic characteristics, by follow-up.

\begin{tabular}{|c|c|c|c|c|c|}
\hline \multicolumn{2}{|c|}{ Socio-demographic characteristics } & \multicolumn{4}{|c|}{ Follow-up group } \\
\hline Number of patients & $\begin{array}{l}N=168 \\
(\%)\end{array}$ & $\begin{array}{l}\text { DIAB } \\
\mathrm{N}=81(48.2)\end{array}$ & $\begin{array}{l}\text { GP } \\
\mathrm{N}=33(19.6)\end{array}$ & $\begin{array}{l}\mathrm{C} 1 \\
\mathrm{~N}=15(8.9)\end{array}$ & $\begin{array}{l}\mathrm{C} 2 \\
\mathrm{~N}=39(23.2)\end{array}$ \\
\hline Male, n (\%) & $123(73.2)$ & $55(67.9)$ & $23(69.7)$ & $12(80)$ & $33(84.6)$ \\
\hline Age, median $\left(\mathrm{iqr}^{1}\right)$ & $62(12.5)$ & $62(11)$ & $60(9)$ & $55(12)$ & $64(14)$ \\
\hline Place of birth Switzerland & $99(58.9)$ & $48(59.2)$ & $27(69.2)$ & $6(40)$ & $26(66.7)$ \\
\hline \multicolumn{6}{|l|}{ Educational level, n (\%) } \\
\hline $\begin{array}{l}\text { Primary school } \\
\text { Secondary school } \\
\text { Professional school } \\
\text { High school } \\
\text { Third-level/university }\end{array}$ & $\begin{array}{l}23(13.7) \\
31(18.4) \\
61(36.3) \\
8(4.8) \\
45(26.8)\end{array}$ & $\begin{array}{l}11(13.6) \\
15(18.5) \\
30(37) \\
6(7.4) \\
19(23.5)\end{array}$ & $\begin{array}{l}2(6.1) \\
9(27.3) \\
13(39.4) \\
1(3) \\
8(24.2)\end{array}$ & $\begin{array}{l}3(20) \\
6(40) \\
2(13.3) \\
0 \\
4(26.7)\end{array}$ & $\begin{array}{l}7(17.9) \\
1(2.6) \\
16(41) \\
1(2.6) \\
14(35.9)\end{array}$ \\
\hline \multicolumn{6}{|c|}{ Annual household income ${ }^{\star}, \mathrm{n}(\%)$} \\
\hline $\begin{array}{l}<20.000 \mathrm{CHF} \\
20.000-30.000 \mathrm{CHF} \\
30.000-50.000 \mathrm{CHF} \\
>50.000 \mathrm{CHF} \\
\text { I don't know } \\
\text { I refuse to answer }\end{array}$ & $\begin{array}{l}11(6.5) \\
23(13.7) \\
32(19) \\
83(49.4) \\
14(8.3) \\
5(3) \\
\end{array}$ & $\begin{array}{l}5(6.2) \\
6(7.4) \\
14(17.3) \\
44(54.3) \\
8(9.9) \\
4(4.9) \\
\end{array}$ & $\begin{array}{l}3(9.1) \\
9(27.3) \\
7(21.2) \\
12(36.4) \\
1(3) \\
1(3) \\
\end{array}$ & $\begin{array}{l}1(6.7) \\
5(33.3) \\
4(26.7) \\
4(26.7) \\
1(6.7) \\
0\end{array}$ & $\begin{array}{l}2(5.1) \\
3(7.7) \\
7(17.9) \\
23(59) \\
4(10.3) \\
0\end{array}$ \\
\hline \multicolumn{6}{|l|}{ Job, n (\%) } \\
\hline $\begin{array}{l}\text { Student (e)/apprentice } \\
\text { Employee } \\
\text { Unemployed/disability } \\
\text { Retired } \\
\text { Housewife/househusband }\end{array}$ & $\begin{array}{ll}0 & \\
58 & (34.5) \\
44 & (26.2) \\
63 & (37.5) \\
3 & (18)\end{array}$ & $\begin{array}{l}0 \\
30(37) \\
15(18.5) \\
35(43.2) \\
1(1.2)\end{array}$ & $\begin{array}{l}0 \\
7(21.2) \\
16(48.5) \\
8(24.2) \\
2(6.1)\end{array}$ & $\begin{array}{l}0 \\
6(40) \\
5(33.3) \\
4(26.7) \\
0\end{array}$ & $\begin{array}{l}0 \\
15(38.5) \\
8(20.5) \\
16(41) \\
0\end{array}$ \\
\hline
\end{tabular}

Iqr $^{1}=$ interquantile range of the median. ${ }^{\star}$ Poverty threshold definition according to the definition currently applied in Switzerland: $<2200$

$\mathrm{CHF} / \mathrm{month}=<26400 \mathrm{CHF} / \mathrm{year}$ for a person living alone. 
Table 2b: Patient's parameters related to diabetes or to metabolic syndrome, depressive and anxiety disorders characteristics, by follow-up $(\mathrm{N}=168)$.

\begin{tabular}{|c|c|c|c|c|c|}
\hline \multicolumn{2}{|c|}{ Parameters related to diabetes or metabolic syndrome characteristics } & \multicolumn{4}{|c|}{ Follow-up group } \\
\hline Number of patients & $\mathrm{N}=168(\%)$ & $\begin{array}{l}\text { DIAB } \\
\mathrm{N}=81(48.2)\end{array}$ & $\begin{array}{l}\text { GP } \\
N=33(19.6)\end{array}$ & $\begin{array}{l}\mathrm{C} 1 \\
\mathrm{~N}=15(8.9)\end{array}$ & $\begin{array}{l}\mathrm{C} 2 \\
\mathrm{~N}=39(23.2)\end{array}$ \\
\hline Duration of type 2 diabetes Years, median (iqr ${ }^{1}$ ) & $11(10)$ & $13(12)$ & $7(9)$ & $7(9)$ & $14(9)$ \\
\hline \multicolumn{6}{|l|}{ Diabetes and metabolic syndrome management } \\
\hline Insulin, n (\%) & $104(61.9)$ & $56(69.1)$ & $6(18.2)$ & $9(60)$ & $33(84.6)$ \\
\hline Total number of medicines, median (iqr $\left.{ }^{1}\right)$ & $5(2)$ & $5(2)$ & $4(2)$ & $6(3)$ & $6(2)$ \\
\hline \multicolumn{6}{|l|}{ Diabetes-related complications, median (iqr $\left.{ }^{1}\right)$} \\
\hline $\begin{array}{l}\text { Macrovascular complications } \\
\text { Microvascular complications } \\
\text { Mixed complications (macrovascular and } \\
\text { microvascular) }\end{array}$ & $\begin{array}{l}0(1) \\
1(2) \\
0\end{array}$ & $\begin{array}{l}0(1) \\
1(2) \\
0\end{array}$ & $\begin{array}{l}0(1) \\
0(1) \\
0\end{array}$ & $\begin{array}{l}0(1) \\
1(3) \\
0\end{array}$ & $\begin{array}{l}0(1) \\
1(2) \\
0\end{array}$ \\
\hline Total vascular complications & $1(2)$ & $1(1)$ & $1(1)$ & $2(2)$ & $2(1)$ \\
\hline $\begin{array}{l}\text { A1C: } \%(\mathrm{mmol} / \mathrm{mol}) \\
\text { median }\left(\mathrm{iqr}^{1}\right)\end{array}$ & $\begin{array}{l}7.15(58) \\
(1.5)\end{array}$ & $\begin{array}{l}7.25(56) \\
(1.8)\end{array}$ & $\begin{array}{l}7.1(54) \\
(1.2)\end{array}$ & $\begin{array}{l}7.1(54) \\
(1.4)\end{array}$ & $\begin{array}{l}7(53) \\
(1.6)\end{array}$ \\
\hline Systolic arterial pressure, $\mathrm{mmHg}$, median $\left(\mathrm{iqr}^{1}\right)$ & $132(22)$ & $131(22)$ & $133(22)$ & $133.5(19)$ & $131.5(27.5)$ \\
\hline Diastolic arterial pressure, $\mathrm{mmHg}$, median $\left(\mathrm{iqr}^{1}\right)$ & $79(14)$ & $79(14)$ & $79.5(13.5)$ & $82(8)$ & $78(16)$ \\
\hline $\begin{array}{l}\text { Urinary albumin/urinary creatinine ratio } \mathrm{mg} / \\
\mathrm{mmol} \text {, median }\left(\mathrm{iqr}^{1}\right)\end{array}$ & $1.4(5.15)$ & $1.4(4.4)$ & $1.36(5.1)$ & $7.3(10.8)$ & $1.15(2.5)$ \\
\hline $\begin{array}{l}\text { Total cholesterol, mg/dl (mmol/l) } \\
\text { median }\left(\text { iqr }^{1}\right)\end{array}$ & $\begin{array}{l}170.1(4.4) \\
(1.35)\end{array}$ & $\begin{array}{l}166.3(4.3) \\
(1.5)\end{array}$ & $\begin{array}{l}185.6(4.8) \\
(1.5)\end{array}$ & $\begin{array}{l}150.8(3.9) \\
(1.2)\end{array}$ & $\begin{array}{l}166.3(4.3) \\
(1)\end{array}$ \\
\hline $\begin{array}{l}\text { LDL-cholesterol, mg/dl (mmol/l) } \\
\text { median }\left(\text { iqr }^{1}\right)\end{array}$ & $\begin{array}{l}85.1(2.2) \\
(1)\end{array}$ & $\begin{array}{l}77.3(2) \\
(1.1)\end{array}$ & $\begin{array}{l}98.6(2.55) \\
(1.2)\end{array}$ & $\begin{array}{l}83.1(2.15) \\
(1)\end{array}$ & $\begin{array}{l}83.1(2.15) \\
(1)\end{array}$ \\
\hline \multicolumn{6}{|l|}{ Depressive and anxiety disorders characteristics } \\
\hline Major depressive disorder, median (iqr ${ }^{1}$ ) & $6(3.6)$ & $1(1.2)$ & 0 & $2(13.3)$ & $3(7.7)$ \\
\hline Minor depressive disorder, median (iqr ${ }^{1}$ ) & $20(11.9)$ & $7(8.6)$ & $6(18.2)$ & $2(13.3)$ & $5(12.8)$ \\
\hline Panic disorder, median (iqr $\left.{ }^{1}\right)$ & $0(0)$ & 0 & 0 & 0 & 0 \\
\hline $\begin{array}{l}\text { Anxiety disorder other than panic disorder, } \\
\text { median (iqr }{ }^{1} \text { ) }\end{array}$ & $8(4.8)$ & $2(2.5)$ & $3(9.1)$ & $1(6.7)$ & $2(5.1)$ \\
\hline
\end{tabular}

Iqr $^{1}=$ interquantile range of the median.

Table 2c. Patient's diabetes risk perception subscale, by follow-up.

\begin{tabular}{llllll}
\hline Patient's diabetes risk perception & \multicolumn{7}{c}{ Follow-up group } \\
\hline Risk perception subscales & $\begin{array}{l}\text { N=168(\%) } \\
\text { median (iqr) }\end{array}$ & $\begin{array}{l}\text { DIAB } \\
\text { N=81(48.2) } \\
\text { median (iqr) }\end{array}$ & $\begin{array}{l}\text { GP } \\
\mathbf{N}=33(19.6) \\
\text { median (iqr) }\end{array}$ & $\begin{array}{l}\text { C1 } \\
\text { N=15(8.9) } \\
\text { median (iqr) }\end{array}$ & $\begin{array}{l}\text { C2 } \\
\text { median (iqr) } \\
\text { median }\end{array}$ \\
\hline $\begin{array}{lllll}\text { Perception of control over diabetes } \\
\text { Diabetes-related worry }\end{array}$ & $3.25(0.5)$ & $3.25(0.5)$ & $2.25(0.5)$ & $3(1)$ & $3.25(0.5)$ \\
Optimistic bias & $2.5(1)$ & $2.5(1)$ & $2.5(1)$ & $3(1)$ & $3(1.5)$ \\
$\begin{array}{l}\text { Perception of the risk related to the } \\
\text { patient's disease (range 1-5) }\end{array}$ & $2(0.5)$ & $2(0.5)$ & $2.5(0.5)$ & $2(1)$ & $2(1)$ \\
$\begin{array}{l}\text { Diabetes risk knowledge (range 0-5) } \\
\text { Environment risk perception }\end{array}$ & $5(1)$ & $2.44(0.9)$ & $2.55(0.9)$ & $2.77(0.8)$ & $2.44(0.9)$ \\
Composite risk perception & $1.66(1)$ & $5(1)$ & $5(1)$ & $5(1)$ & $5(1)$ \\
\hline All & $2.21(0.6)$ & $2.14(0.5)$ & $2.26(0.5)$ & $2.67(0.2)$ & $2.25(0.6)$ \\
\hline
\end{tabular}

All subscales are in range $1(\min )-4(\max )$ except where noted differently.

Factors related to diabetes or to metabolic syndrome The median (iqr) age diabetes duration was 11(10) years, and over half suffered from microvascular complications (68.3\% men and $53.3 \%$ women), but had fewer macrovascular complications overall (37.3\%). Glycated haemoglobin, blood pressure, kidney function, albuminuria, and lipid profile values at the time of the study demonstrated very good disease control.

\section{Psychological characteristics}

In terms of psychological profiles by the PRIME-MD/PHQ: 3.6\% 
exhibited symptoms of a major depressive disorder, $11.9 \%$ had a minor depressive disorder, and $4.8 \%$ had an anxiety disorder (other than panic disorder).

\section{Diabetes risk perception subscales}

Patients had low median levels of perceived risk related to their disease (2.44), diabetes-related worry (2.5), optimistic bias (2), environment risk perception (1.66), and a low median composite risk perception (2.21). Patients had a high median level of perception of control over diabetes (3.25) and a very high perception of knowledge regarding risk (5).

\section{Diabetes risk perception subscales according to different follow-up}

Patients of the $\mathrm{C} 1$ group, compared to the DIAB group, had higher perception of risk related to their disease (median level: 2.77 versus 2.44). These patients, compared to the DIAB group also had more diabetes-related worry (median level 3 versus 2.5) and a higher level of composite risk perception (median level 2.67 versus 2.14).

\section{Logistic regression analysis of perceived risk}

Among 168 patients, $138(82.1 \%)$ had a higher perception of control over diabetes, 80 (47.6\%) had higher diabetes-worry, 36 (21.4\%) had a higher optimistic bias, 39 (23.2\%) had a higher perception of the risk related to diabetes complications, 146 (86.9\%) had a higher diabetes risk knowledge, 31 (18.4\%) had a higher environment risk perception and 52 (30.9\%) had a higher composite risk perception.

In the univariate analysis in logistic regression (Tables 3a-3c), patients with combined specialty and general practitioner follow-up (C1), compared to the DIAB follow-up, had

Table 3a: Univariate logistic regression - type of follow-up, sociodemographic characteristics and diabetes risk perception subscales.

\begin{tabular}{|c|c|c|c|c|c|c|c|}
\hline \multirow[t]{2}{*}{${ }^{\star}$ Types of follow-up } & \multicolumn{7}{|c|}{ Diabetes risk perception subscales (coded in two categories: low $=0$ vs high $=1)$ OR $(p)$} \\
\hline & $\begin{array}{l}\text { Perception of } \\
\text { control over } \\
\text { diabetes }\end{array}$ & $\begin{array}{l}\text { Diabetes- } \\
\text { related } \\
\text { worry }\end{array}$ & $\begin{array}{l}\text { Optimistic } \\
\text { bias }\end{array}$ & $\begin{array}{l}\text { Perception of the } \\
\text { risk related to the } \\
\text { patient's disease }\end{array}$ & $\begin{array}{l}\text { Diabetes risk } \\
\text { knowledge }\end{array}$ & $\begin{array}{l}\text { Environment } \\
\text { risk perception }\end{array}$ & $\begin{array}{l}\text { Composite risk } \\
\text { perception }\end{array}$ \\
\hline GP & $0.71(0.49)$ & $1.34(0.48)$ & $1.12(0.82)$ & $0.90(0.85)$ & $0.42(0.15)$ & $1.67(0.31)$ & $1.31(0.57)$ \\
\hline $\mathrm{C} 1$ & $0.62(0.47)$ & $6.45(0.006)$ & $* * *$ & $3.55(0.03)$ & $0.38(0.20)$ & $1.90(0.33)$ & $14(<0.0001)$ \\
\hline $\mathrm{C} 2$ & $2.72(0.13)$ & $2.09(0.06)$ & $1.21(0.68)$ & $1.40(0.46)$ & $0.52(0.27)$ & $0.95(0.93)$ & $1.75(0.19)$ \\
\hline $\begin{array}{l}\text { Socio demographic } \\
\text { characteristics }\end{array}$ & \multicolumn{7}{|c|}{ Diabetes risk perception subscales OR (p) } \\
\hline Male & $0.99(0.99)$ & $1.19(0.62)$ & $0.94(0.88)$ & $1.90(0.16)$ & $0.78(0.65)$ & $1.32(0.56)$ & $1.32(0.47)$ \\
\hline Age & $\begin{array}{l}0.95 \\
(0.03)\end{array}$ & $\begin{array}{l}0.97 \\
(0.04)\end{array}$ & $\begin{array}{l}1.04 \\
(0.048)\end{array}$ & $\begin{array}{l}1.02 \\
(0.35)\end{array}$ & $\begin{array}{l}0.95 \\
(0.05)\end{array}$ & $\begin{array}{l}0.98 \\
(0.23)\end{array}$ & $\begin{array}{l}0.98 \\
(0.19)\end{array}$ \\
\hline Place of birth & & & & & & & \\
\hline Outside of Switzerland & $0.76(0.49)$ & $2.03(0.03)$ & $0.77(0.5)$ & $1(0.99)$ & $1.26(0.63)$ & $1.04(0.91)$ & $1.70(0.12)$ \\
\hline \multicolumn{8}{|l|}{ Educational level } \\
\hline $\begin{array}{l}\text { Primary school }^{* *} \\
\text { Secondary school } \\
\text { Professional school } \\
\text { High school } \\
\text { Third-level/university }\end{array}$ & $\begin{array}{l}0.88(0.85) \\
1.39(0.62) \\
\star * * \\
0.58(0.40)\end{array}$ & $\begin{array}{l}0.5(0.22) \\
0.37(0.05) \\
0.89(0.89) \\
0.43(0.11)\end{array}$ & $\begin{array}{l}0.53(0.39) \\
1.17(0.78) \\
\star * * \\
1.31(0.66)\end{array}$ & $\begin{array}{l}0.83(0.77) \\
0.84(0.76) \\
0.40(0.44) \\
0.92(0.88)\end{array}$ & $\begin{array}{l}1.01(0.99) \\
1.16(0.84) \\
1.05(0.97) \\
0.81(0.78)\end{array}$ & $\begin{array}{l}0.86(0.83) \\
0.54(0.33) \\
0.51(0.57) \\
1.16(0.35)\end{array}$ & $\begin{array}{l}0.89(0.85) \\
0.61(0.35) \\
1.12(0.89) \\
1.03(0.95)\end{array}$ \\
\hline \multicolumn{8}{|c|}{ Annual household income } \\
\hline $\begin{array}{l}<20.000 \mathrm{CHF}^{* *} \\
20.000-30.000 \mathrm{CHF} \\
30.000-50.000 \mathrm{CHF} \\
>50.000 \mathrm{CHF} \\
\text { I don't know } \\
\text { I refuse to answer }\end{array}$ & $\begin{array}{l}1.35(0.72) \\
0.82(0.80) \\
3.51(0.10) \\
1.37(0.73) \\
1.5(0.76)\end{array}$ & $\begin{array}{l}0.44(0.28) \\
1.22(0.78) \\
0.74(0.64) \\
0.83(0.82) \\
0.21(0.22)\end{array}$ & $\begin{array}{l}1.5(0.74) \\
2.8(0.36) \\
3.4(0.26) \\
4(0.25) \\
* * \star\end{array}$ & $\begin{array}{l}0.74(0.72) \\
0.49(0.4) \\
0.85(0.82) \\
1.48(0.65) \\
0.67(0.76)\end{array}$ & $\begin{array}{l}1.05(0.95) \\
1.2(0.84) \\
1.45(0.66) \\
* * * \\
* * *\end{array}$ & $\begin{array}{l}0.12(0.08) \\
0.75(0.71) \\
0.69(0.61) \\
0.73(0.73) \\
* * *\end{array}$ & $\begin{array}{l}1.17(0.85) \\
1.4(0.66) \\
1.1(0.91) \\
2(0.42) \\
0.67(0.76)\end{array}$ \\
\hline \multicolumn{8}{|l|}{ Job } \\
\hline $\begin{array}{l}\text { Student/apprentice } \\
\text { Employee }\end{array}$ & $* * *$ & $* * *$ & $* * *$ & $* * *$ & $* * *$ & $* * *$ & $* * *$ \\
\hline Unemployed/disable & $0.84(0.76)$ & $0.81(0.6)$ & $0.81(0.69)$ & $1.26(0.64)$ & $0.9(0.88)$ & $0.55(0.26)$ & $1.06(0.89)$ \\
\hline Retired & $0.51(1.16)$ & $0.57(0.13)$ & $1.45(0.40)$ & $1.71(0.22)$ & $0.54(0.26)$ & $0.73(0.5)$ & $0.82(0.62)$ \\
\hline Housewife/-bound & $* * *$ & $* * *$ & $8.54(0.09)$ & $* * *$ & $* * *$ & $1.73(0.66)$ & $* * *$ \\
\hline
\end{tabular}

${ }^{\star} \mathrm{DIAB}=$ reference group, ${ }^{* *}=$ reference group,${ }^{* *}=$ no patients in this group (perfect prediction) 
Table 3b. Univariate logistic regression - parameters related to diabetes and metabolic syndrome characteristicsand diabetes risk perception subscales.

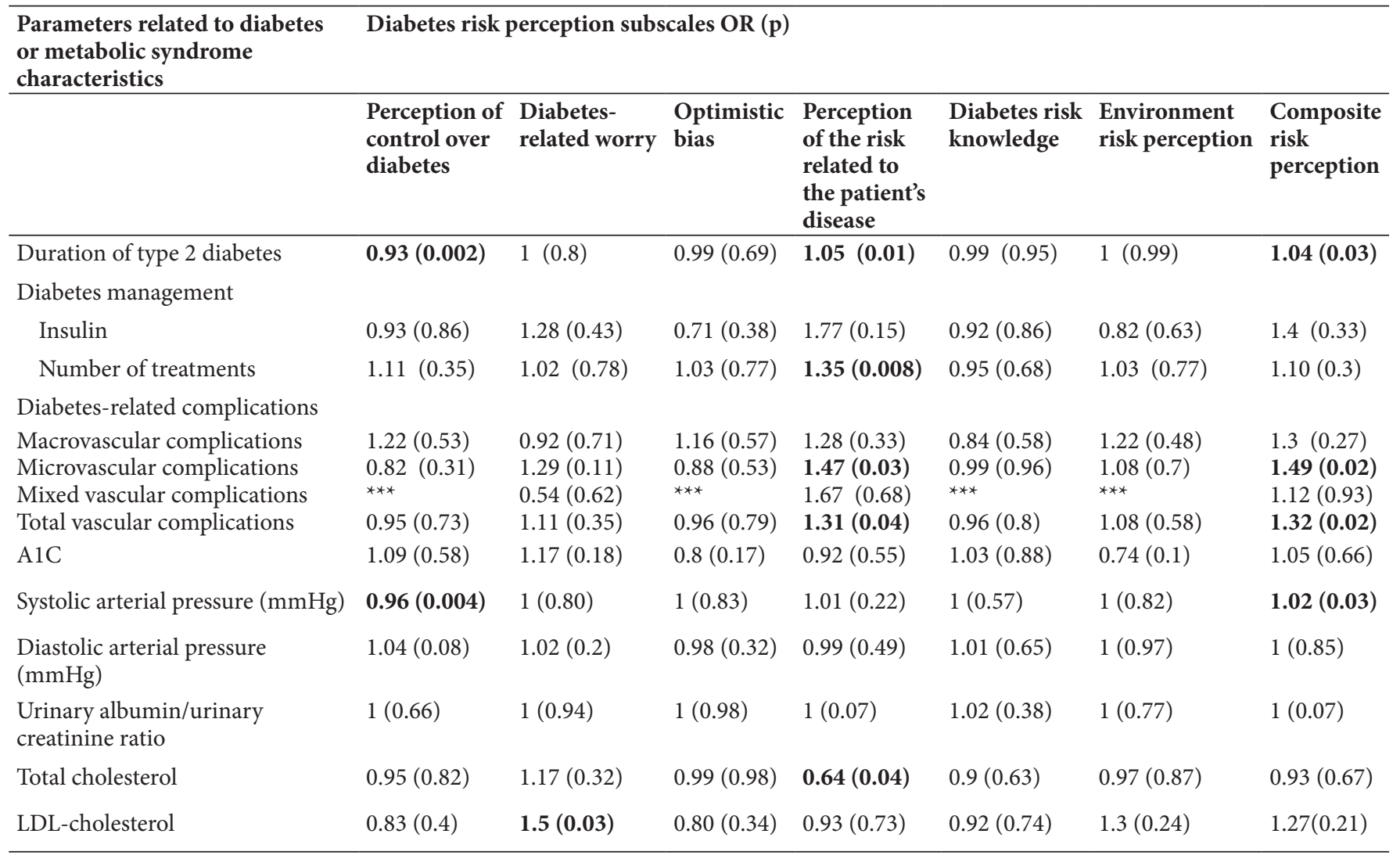

${ }^{*} \mathrm{DIAB}=$ reference group, ${ }^{* *}=$ reference group,${ }^{* *}=$ no patients in this group (perfect prediction)

Table 3c. Univariate logistic regression-depressive and anxiety disorders characteristics and diabetes risk perception subscales.

\begin{tabular}{|c|c|c|c|c|c|c|c|}
\hline \multirow{2}{*}{$\begin{array}{l}\text { Depressive and anxiety } \\
\text { disorders characteristics }\end{array}$} & \multicolumn{7}{|c|}{ Diabetes risk perception subscales OR (p) } \\
\hline & $\begin{array}{l}\text { Perception of } \\
\text { control over } \\
\text { diabetes }\end{array}$ & $\begin{array}{l}\text { Diabetes- } \\
\text { related worry }\end{array}$ & $\begin{array}{l}\text { Optimistic } \\
\text { bias }\end{array}$ & $\begin{array}{l}\text { Perception of } \\
\text { the risk related } \\
\text { to the patient's } \\
\text { disease }\end{array}$ & $\begin{array}{l}\text { Diabetes } \\
\text { risk } \\
\text { knowledge }\end{array}$ & $\begin{array}{l}\text { Environment } \\
\text { risk } \\
\text { perception }\end{array}$ & $\begin{array}{l}\text { Composite } \\
\text { risk } \\
\text { perception }\end{array}$ \\
\hline Major depressive disorder & $* * *$ & $* * *$ & $0.72(0.77)$ & $0.65(0.70)$ & $* * *$ & $0.88(0.91)$ & $2.3(0.32)$ \\
\hline Minor depressive disorder & $2.1(0.34)$ & $1.4(0.48)$ & $0.61(0.046)$ & $0.81(0.72)$ & $0.4(0.10)$ & $* * *$ & $0.95(0.92)$ \\
\hline $\begin{array}{l}\text { Anxiety disorder other than } \\
\text { panic disorder }\end{array}$ & $0.34(0.15)$ & $8.3(0.05)$ & $* * *$ & $1.11(0.90)$ & $0.43(0.32)$ & $0.62(0.66)$ & $7.43(0.02)$ \\
\hline
\end{tabular}

${ }^{\star} \mathrm{DIAB}=$ reference group, ${ }^{* *}=$ reference group,${ }^{* *}=$ no patients in this group (perfect prediction)

higher composite risk perception $(\mathrm{OR}=14 ; 95 \% \mathrm{Cl} 3.55-55.06$; $\mathrm{p}=<0.0001)$, higher diabetes-worry $(\mathrm{OR}=6.45 ; 95 \% \mathrm{Cl} 1.68-24.7$; $\mathrm{p}=0.006)$ and higher risk perception for diabetes complications $(\mathrm{OR}=3.55 ; 95 \% \mathrm{Cl} 1.12-11.25 ; \mathrm{p}=0.03)$. Older age was associated with lower perceived control over diabetes $(\mathrm{OR}=0.95$; 95\% Cl 0.91-0.99; $p=0.03)$, lower diabetes-related worry $(\mathrm{OR}=0.97 ; 95 \% \mathrm{Cl} 0.94-1 ; \mathrm{p}=0.04)$, higher odds of optimistic bias $(\mathrm{OR}=1.04 ; 95 \% \mathrm{Cl} 1-1.8 ; \mathrm{p}=0.048)$ and lower diabetes risk knowledge $(\mathrm{OR}=0.95 ; 95 \% \mathrm{Cl} 0.91-1 ; \mathrm{p}=0.05)$. Increased duration of diabetes was associated with lower odds of perceived control of diabetes $(\mathrm{OR}=0.93 ; 95 \% \mathrm{Cl} 0.89-0.97 ; \mathrm{p}=0.002)$, but higher odds of perceived risk related to the patient's illness $(\mathrm{OR}=1.05 ; 95 \% \mathrm{Cl} 1.01-1.09 ; \mathrm{p}=0.012)$, and the composite perceived risk $(\mathrm{OR}=1.04 ; 95 \% \mathrm{Cl} 1-1.08 ; \mathrm{p}=0.03)$. Specific microvascular complications and total vascular complications were positively correlated with perceived risk related 
to the patient's illness $(\mathrm{OR}=1.47 ; 95 \% \mathrm{Cl} 1.03-2.10 ; \mathrm{p}=0.03$ and $\mathrm{OR}=1.31 ; 95 \% \mathrm{Cl} 1.08-1.69 ; \mathrm{p}=0.04)$ and composite perceived risk $(\mathrm{OR}=1.49 ; 95 \% \mathrm{Cl} 1.07-2.09 ; \mathrm{p}=0.02$ and $\mathrm{OR}=1.32$; $95 \% \mathrm{Cl} 1.04-1.69 ; \mathrm{p}=0.02)$. Having an anxiety disorder other than panic disorder increased the odds for diabetes related worry $(\mathrm{OR}=8.3 ; 95 \% \mathrm{Cl} 1-69.4 ; \mathrm{p}=0.05)$ and a higher composite perceived risk score $(\mathrm{OR}=7.43 ; \mathrm{Cl} 95 \% 1.45-39.19 ; \mathrm{p}=0.02)$.

In the multivariate analysis (Table 4), there were no differences between the type of providers and the perception of control of diabetes. However C1 group (compared to DIAB group) had higher levels for worry of diabetes (OR 5.73; 95\% CI 1.42-23.07; $\mathrm{p}=0.014$ ), increased perceived risk related to the patient's illness (OR 4.43; 95\% Cl 1.26-15.6; $p=0.02)$ and better composite risk perception (OR $21 ; 95 \% \mathrm{Cl}$ 4.93-89.61; $\mathrm{p}=0$ ). For the three other subscales, no model for a multivariate analysis was possible.

\section{Discussion}

\section{Perceived risk of diabetes by follow-up type}

A significantly higher composite risk perception was associated with the combined follow-up group, when compared to all other groups. This was also true for perceived risk for diabetes complications and diabetes-related worry in rela-

Table 4. Multivariate logistic regression analysis of some diabetes risk subscales adjustable to some patient characteristics or types of follow-up.

\begin{tabular}{|c|c|}
\hline \multicolumn{2}{|l|}{ Perception of control over diabetes } \\
\hline Duration of type 2 diabetes & $0.93(0.003)$ \\
\hline Systolic arterial pressure (mmHg, median) & $0.97(0.05)$ \\
\hline \multicolumn{2}{|l|}{ Type of follow-up (DIAB = reference group) } \\
\hline GP & $0.37(0.10)$ \\
\hline $\mathrm{C} 1$ & $0.29(0.09)$ \\
\hline $\mathrm{C} 2$ & $2.2(0.27)$ \\
\hline \multicolumn{2}{|l|}{ Diabetes-related worry } \\
\hline Age & $0.96(0.048)$ \\
\hline LDL-cholesterol (mmol/l, median) & $1.5(0.042)$ \\
\hline \multicolumn{2}{|l|}{ Type of follow-up (DIAB = reference group) } \\
\hline GP & $0.99(0.98)$ \\
\hline $\mathrm{C} 1$ & $5.73(0.014)$ \\
\hline $\mathrm{C} 2$ & $1.66(0.24)$ \\
\hline \multicolumn{2}{|c|}{ Perception of the risk related to the patient's disease } \\
\hline Duration of type 2 diabetes & $1.05(0.017)$ \\
\hline Total number of medications & $1.27(0.054)$ \\
\hline \multicolumn{2}{|l|}{ Type of follow-up (DIAB = reference group) } \\
\hline GP & $1.68(0.37)$ \\
\hline $\mathrm{C} 1$ & $4.43(0.02)$ \\
\hline $\mathrm{C} 2$ & $1.25(0.63)$ \\
\hline \multicolumn{2}{|l|}{ Composite risk perception } \\
\hline Duration of type 2 diabetes & $1.06(0.005)$ \\
\hline Anxiety disorder other than panic disorder & $8.83(0.015)$ \\
\hline \multicolumn{2}{|l|}{ Type of follow-up (DIAB = reference group) } \\
\hline GP & $1.76(0.29)$ \\
\hline $\mathrm{C} 1$ & $21(0)$ \\
\hline $\mathrm{C} 2$ & $1.72(0.24)$ \\
\hline
\end{tabular}

tion to combined follow-up. These results show that being followed by several healthcare professionals who all work in the same centre (such as our polyclinic) increases/improves the perceived risk of this disease.

Improved metabolic control of diabetes has been documented within the context of an intensive team approach to diabetes management $[12,13]$. Intensive management of diabetes constitutes far more than an increased frequency of monitoring or an additional injection of insulin per day. It involves careful follow-up as well as an evaluation of the medical, educational and psychosocial needs of the patient. Diabetic patients manage their illness on a daily basis within the wider context of other goals, priorities, health issues and other personal concerns that make up their lives. The behaviours of these patients are often affected by multiple variables, as the kind of patient-physician relationship, complexity of regimen, disruption of lifestyle, emotional support, financial resources and education in self-management skills. These findings highlight the need to develop ways of empowering patients within the context of multidisciplinary care [22].

Diabetes is associated with many comorbidities and challenging lifestyle recommendations. Consequently, it is difficult for any single physician to treat all of them in a single consultation. An interdisciplinary care model, like that of our polyclinic, offers goal-directed patient-centred care, with equal emphasis placed on education, counselling and medical treatment [13]. Integrated care shares provider responsibility while all members address common patientprovider goals, and collaborate with, rather than just consult one another. It requires a unified team and the adoption of a shared professional identity with a marked team spirit as well as a common objective. A multidisciplinary team approach is the best way to manage diabetes, as shown by the results of multiple studies; medication alone is not enough to reduce diabetes complications and a multidisciplinary team approach to the management of diabetes is the most effective way to achieve better glycaemic control, improve blood pressure and lipid levels [23]. Many studies also show that a multidisciplinary approach to diabetes improves quality of life, decreases health costs, lowers risk of complications, increases follow-up and promotes higher patient satisfaction [24]. A combined follow-up in the same setting is also likely to facilitate the patient's ability to access essential resources and care at any given time, which is probably linked to better knowledge and increased risk perception. This is likely because each member of a larger interdisciplinary team can offer complementary management skills, more individual time for the assessment and treatment of patients. The group offers a more complete care model and facilitates stronger provider-patient foundations. Such teams assist patients in encouraging them to learn diabetes self-care through consistent messages, repetition of information, reinforcement and feedback of self-care behaviours [25]. However, regular and ongoing communication among team members is necessary 
for an effective team to function [25].

Diabetes risk subscales and their associations with patients' characteristics, metabolic profile and psychological profile

Overall, composite risk perception was fairly low (2.27, range 1-4, low level: 1-2.49), indicating that these patients have a low global diabetes risk perception. Patients also had low risk perception for diabetes complications (2.52, range 1-5, low level: 1-2.99). However, factors related to more complex disease (longer diabetes duration, increased number of drug therapies, increased microvascular and total vascular complications) were correlated with a higher perceived risk for diabetes complications. Interestingly, we did not find any studies that showed and explained why higher disease burden was associated with higher perceived risk.

The patients in our study displayed low optimistic bias (mean score: 2.24, range 1-4, low level: 1-2.99). Research in this area indicates that optimistic bias is lower in people with a higher level of education $[10,19]$ and in people who were born in their country of residence [1]. Among people at risk of developing diabetes, men display more optimistic bias [11], which decreases if the epidemiological risk of developing the disease is greater [12]. Despite the absence of a statistically significant correlation in our study, the low optimistic bias may be accounted for by a good overall level of education, and coupled with better (higher) perception of the actual risk. Older age was significantly associated with greater optimistic bias (OR=1.04;95\% Cl 1-1.8; $\mathrm{p}=0.048)$; the literature on this subject offers no clear explanation for this observation, but it has been shown that the elderly have a poorer perception of the risk of developing diabetes, a possible result of greater optimistic bias [13-16].

Finally, diabetes-related worry was low (mean score 2.66, range 1-4, low level: 1-2.99), and was significantly associated with the presence of any anxiety disorder except panic disorder $(\mathrm{OR}=8.3 ; 95 \% \mathrm{Cl} 1-69.4 ; \mathrm{p}=0.05)$. Greater diabetes-related worry can be advantageous as it heightens risk perception, but if worry is excessive, it can be debilitating, and this anxiety can lower quality of life. For example, it has been documented that excessive worry narrows the patient's field of perception to the present cause of stress, reduces cognitive capacity (ability to elaborate on information about their health) and prevents patients from taking a more active part in their disease management $[17,18]$.

\section{Diabetes risk perception subscales compared to original RPS-DM study}

Our study population had higher risk perception for most of the subscales, and higher overall values compared to those in Walker's findings for the RPS-DM questionnaire in an urban study population [1]. Our results show higher perception of control over diabetes (3.23/2.9, range 1-4), less optimistic bias (2.24/2.4, range 1-4) and higher knowledge of diabetes risk (4.33/3.7, range 0-5). Diabetes-related worry was lower than the Walker study (2.66/3.1, range 1-4).

In our investigation (unlike in E.A. Walker's study), no statistically significant correlation was found between level of education and the risk perception subscales. The overall good level of education of our patients may explain this result.

\section{Strengths and limitations}

To our knowledge, this is the first study to evaluate the effects of combined care on risk perception among patients with diabetes. We examined associations between subscales of diabetes risk perception and socio-demographic characteristics similar to a large previous study [1]. However, the current study also investigated the associations of risk perception with metabolic syndrome and psychological characteristics of patients, an added improvement on previous work in this area.

The advantages of a combined follow-up, especially when in the same institution, are probably the best explanation of this increased risk perception, but other factors could interfere with this result; patients of the $\mathrm{C} 1$ group, compared to patients of the DIAB group, had lower socio-economic status (within this group unemployment is higher and their annual income is, on the whole, lower), are younger and with a younger diabetes and parts of these factors could influence some aspects of risk perception [1].

Interestingly, we did not find a better risk perception of diabetes among patients who had a combined follow-up with physicians who do not practice in the same institution (C2 group) compared to patients of the DIAB group. This could be explained by a possible difficulty to provide patients with a coherent health message as well as offering a coordinated care as with a combined follow-up in the same institution.

Since this study is cross-sectional, it is not possible to establish the direction of the relationship between type follow-up and diabetes risk perception. The patients in each group may be highly self-selected, thus there may be reverse causation as well: the patients with higher risk perception may choose different follow-up methods, or doctors may be more likely to refer them differently.

Due to the lack of power of our study (mainly for the combined follow-up group), it was not possible to analyse whether patients' characteristics associated with type of follow-up were statistically relevant and, clinically, had a real impact on different risk perception according to type of follow-up.

The small number of patients included constitutes one of the major limitations of our study. Many non-French speakers, patients from the polyclinic, were excluded based on linguistic competence. Furthermore, the population of this institution (serving a significant portion of the underserved in the area) does not accurately reflect the socio-demographic reality of the entire population of Lausanne. Finally, due to the low turnout rate of participants, our results should be interpreted with caution. Despite a better/higher risk perception of diabetes, patients in the combined follow-up group, did 
not have a better biological profile or lower blood pressure values compared to other groups; however, it was difficult to detect any small differences in disease profile given the small size of the comparison groups, and the overall good disease profile for the study population, in general.

\section{Conclusion}

To our knowledge, this is the first study to analyse several domains of diabetes risk perception with "a validated French" version of E.A. Walker's RPS-DM survey [1]. Our results suggest that the collaboration by several healthcare professionals (diabetologists and general practitioners) who work at the same centre may improve their patients' diabetes risk perception. Annual income, age, diabetes duration, the number of vascular complications, and the presence of any anxiety disorder other than panic disorder were the factors most significantly associated with higher diabetes risk perception subscales.

Given that this is a pilot study, the next steps include a multi-centric longitudinal study examining these relationships among different provider group types. Future research should also better explore the link between perception of diabetes complications and a more severe disease.

\section{Competing interests}

The authors declare that they have no competing interests.

Authors' contributions

\begin{tabular}{|l|l|l|l|l|l|}
\hline Authors' contributions & FG & PB & JR & AC & FM \\
\hline Research concept and design & $\checkmark$ & $\checkmark$ & $\checkmark$ & $\checkmark$ & $\checkmark$ \\
\hline Collection and/or assembly of data & $\checkmark$ & -- & -- & -- & -- \\
\hline Data analysis and interpretation & $\checkmark$ & $\checkmark$ & $\checkmark$ & $\checkmark$ & $\checkmark$ \\
\hline Writing the article & $\checkmark$ & $\checkmark$ & -- & $\checkmark$ & $\checkmark$ \\
\hline Critical revision of the article & $\checkmark$ & $\checkmark$ & $\checkmark$ & $\checkmark$ & $\checkmark$ \\
\hline Final approval of article & $\checkmark$ & $\checkmark$ & $\checkmark$ & $\checkmark$ & $\checkmark$ \\
\hline Statistical analysis & -- & -- & -- & -- & $\checkmark$ \\
\hline
\end{tabular}

\section{Acknowledgements}

This study was financially supported by the Endocrinology and Diabetology Service of the Lausanne University Hospital (CHUV), by the Department of Ambulatory Care and Community Medicine of the Lausanne University Hospital (PMU), and by the Swiss National Science Foundation (K-31KO-120554).

Dr. Elizabeth A. Walker, Albert Einstein College of Medicine, Bronx, NY, United States, for her kind permission to use her RPS-DM survey. Ms Johanna Norton, epidemiological statistician, research engineer at the INSERM (French National Institute of Health and Medical Research), Montpellier, France, for her advice and her permission to use her French version of the PHQ.

Ms Lavinia Giacomini, psychologist, Lausanne, Switzerland.

The assistant doctors of the Endocrinology and Diabetology Service of the Lausanne University Hospital (CHUV) and the assistant doctors of the Department of Ambulatory Care and Community Medicine of the Lausanne University Hospital (PMU).

Mrs Jessica Stevens, Nyon, Switzerland.

\section{Publication history}

Editor: Charles Heilig, University of Florida, USA.

Received: 03-July-2017 Final Revised: 19-Sep-2017

Accepted: 23-Oct-2017 Published: 05-Nov-2017

\section{References}

1. Walker EA, Caban A, Schechter CB, Basch CE, Blanco E, DeWitt T, Kalten $\mathrm{MR}$, Mera MS and Mojica G. Measuring comparative risk perceptions in an urban minority population: the risk perception survey for diabetes. Diabetes Educ. 2007; 33:103-10. | Article | PubMed

2. Harris R and Linn MW. Health beliefs, compliance, and control of diabetes mellitus. South Med J. 1985; 78:162-6. | PubMed

3. Claassen L, Henneman L, Nijpels G, Dekker J, Marteau T and Timmermans D. Causal beliefs and perceptions of risk for diabetes and cardiovascular disease, The Netherlands, 2007. Prev Chronic Dis. 2011; 8:A130. | Article | PubMed Abstract | PubMed FullText

4. Walker E, Fisher EB and Marreri DG W.M. Comparative Risk Judgements among participants in the Diabetes Prevention Program. Diabetes Care. 2001; 50:A397.

5. Adriaanse MC, Snoek FJ, Dekker JM, Spijkerman AM, Nijpels G, van der Ploeg HM and Heine RJ. Perceived risk for Type 2 diabetes in participants in a stepwise population-screening programme. Diabet Med. 2003; 20:210-5. | Article | PubMed

6. Frijling $B D$, Lobo $C M$, Keus IM, Jenks KM, Akkermans RP, Hulscher ME, Prins A, van der Wouden JC and Grol RP. Perceptions of cardiovascular risk among patients with hypertension or diabetes. Patient Educ Couns. 2004; 52:47-53. | Article | PubMed

7. Wagner J, Lacey K, Abbott G, de Groot M and Chyun D. Knowledge of heart disease risk in a multicultural community sample of people with diabetes. Ann Behav Med. 2006; 31:224-30. | Article | PubMed

8. Benchmark JC. Perception of risk in diabetes points the vay to patient education. Joint Commission on Accreditation of Healthcare Organizations. 2002; 4:8-9.

9. Eiser JR, Eiser C, Riazi A, Hammersley S and Tooke JE. Interpretations of Risk and Expectations of Change among Individuals with Types 1 and 2 Diabetes. J Health Psychol. 2002; 7:701-11. | Article | PubMed

10. Allen JK, Purcell A, Szanton S and Dennison CR. Perceptions of cardiac risk among a low-income urban diabetic population. J Health Care Poor Underserved. 2010; 21:362-70. | Article | PubMed Abstract | PubMed FullText

11. Asimakopoulou KG, Skinner TC, Spimpolo J, Marsh S and Fox C. Unrealistic pessimism about risk of coronary heart disease and stroke in patients with type 2 diabetes. Patient Educ Couns. 2008; 71:95-101. | Article | PubMed

12. Nathan DM, Genuth S, Lachin J, Cleary P, Crofford O, Davis M, Rand L and Siebert $C$. The effect of intensive treatment of diabetes on the development and progression of long-term complications in insulindependent diabetes mellitus. N Engl J Med. 1993; 329:977-86. | Article I PubMed

13. Bayless Maa. The Team Approach to Intensive Diabetes Management. Diabetes Spectrum. 1998; 11:33-37.

14. Scollan-Koliopoulos M, Walker EA and Bleich D. Perceived risk of amputation, emotions, and foot self-care among adults with type 2 diabetes. Diabetes Educ. 2010; 36:473-82. | Article | PubMed Abstract | PubMed FullText

15. Lustman PJ, Anderson RJ, Freedland KE, de Groot M, Carney RM and Clouse RE. Depression and poor glycemic control: a meta-analytic review of the literature. Diabetes Care. 2000; 23:934-42. | Article | PubMed

16. Slovic. The perception of Risk: introduction and Overview. London, UK: Earthscan. 2000.

17. Slovic P. Perception of risk. Science. 1987; 236:280-5. | Article | PubMed

18. Spitzer RL, Kroenke $K$ and Williams JB. Validation and utility of a selfreport version of PRIME-MD: the PHQ primary care study. Primary Care Evaluation of Mental Disorders. Patient Health Questionnaire. JAMA. 1999; 282:1737-44. | Article | PubMed

19. Colonna RG-D et al. Evaluation des Troubles Affectifs par le Practicien; Guide du Clinicien. Version française du PRIME MD. 1994.

20. Norton J, De Roquefeuil G, Boulenger JP, Ritchie K, Mann A and Tylee A. Use of the PRIME-MD Patient Health Questionnaire for estimating the 
prevalence of psychiatric disorders in French primary care: comparison with family practitioner estimates and relationship to psychotropic medication use. Gen Hosp Psychiatry. 2007; 29:285-93. | Article | PubMed

21. Darlow S, Goodman MS, Stafford JD, Lachance CR and Kaphingst KA. Weight perceptions and perceived risk for diabetes and heart disease among overweight and obese women, Suffolk County, New York, 2008. Prev Chronic Dis. 2012; 9:E81. | Article | PubMed Abstract | PubMed FullText

22. Borgermans L, Goderis G, Van Den Broeke C, Verbeke G, Carbonez A, Ivanova A, Mathieu C, Aertgeerts B, Heyrman J and Grol R. Interdisciplinary diabetes care teams operating on the interface between primary and specialty care are associated with improved outcomes of care: findings from the Leuven Diabetes Project. $B M C$ Health Serv Res. 2009; 9:179. | Article | PubMed Abstract | PubMed FullText

23. Gagliardino JJ and Etchegoyen G. A model educational program for people with type 2 diabetes: a cooperative Latin American implementation study (PEDNID-LA). Diabetes Care. 2001; 24:1001-7. | Article | PubMed

24. Codispoti C, Douglas MR, McCallister T and Zuniga A. The use of a multidisciplinary team care approach to improve glycemic control and quality of life by the prevention of complications among diabetic patients. J Okla State Med Assoc. 2004; 97:201-4. | PubMed

25. Ritholz MD, Beverly EA, Abrahamson MJ, Brooks KM, Hultgren BA and Weinger K. Physicians' perceptions of the type 2 diabetes multidisciplinary treatment team: a qualitative study. Diabetes Educ. 2011; 37:794-800. | Article | PubMed Abstract | PubMed FullText

\section{Citation:}

Gianinazzi F, Ruiz J, Faouzi M, Casillas A and Bodenmann P. Comparing diabetes mellitus risk perception among patients followed in primary and specialized healthcare: A cross-sectional study in Vaud, Switzerland. J Diab Res Clin Met. 2017; 6:1.

http://dx.doi.org/10.7243/2050-0866-6-1 